\title{
The Mozart effect combined with specific kinetic treatment in patients with Parkinson's disease
}

\author{
BREDA Xénia Melánia ${ }^{1}$, FODOR Dana Marieta ${ }^{1}$, VERDEŞ Robert $^{1}$, TĂRTĂMUŞ Dorina ${ }^{2}$, MUREŞAN \\ Dorin-Alexandru ${ }^{3,4}$, STĂNESCU Ioana C. ${ }^{1,3}$, CĂPUŞAN Camelia ${ }^{5}$, DUMBRAVĂ PERJU Lăcrămioara ${ }^{1}$ \\ Corresponding Author: Dana Marieta Fodor, email: fodordana@yahoo.com
} Balneo Research Journal DOI: http://dx.doi.org/10.12680/balneo.2019.271 Vol.10, No.3, September 2019 p: 294-299

1. "Iuliu Haţieganu" University of Medicine and Pharmacy,Cluj Napoca, Romania 2."Puterea Sperantei” Project, Cluj Napoca, Romania

${ }^{3}$ Rehabilitation Hospital Cluj Napoca, Romania 4.Department of Sport and Physical Education Science, Babes - Bolyai University, Cluj-Napoca, Romania 5"LaVitaMed" Clinic, Cluj Napoca, Romania

\begin{abstract}
Introduction \& objectives: Although controversial, the Mozart effect, consisting of an improvement in cognitive abilities in healthy persons, has started to be studied in the case of patients with various neurological, even neurodegenerative diseases (dementia, treatment-refractory epilepsy). There are few data on the Mozart effect in Parkinson's disease, but the positive results reported for other neurological pathologies have led us to study this subject. The aim of this study is to compare the results of the Mozart effect associated with specific kinetic treatment in the rehabilitation of Parkinson's disease and the effect of standard kinesiotherapy, results reflected in the impact on the degree of disability, emotional state and quality of life.
\end{abstract}

Material and method: This was a prospective case-control study, comprising patients with Parkinson's disease, Hoehn \& Yahr stages I-III, included in a motor neurorehabilitation program through the project "The power of hope for patients with Parkinson's disease" Cluj-Napoca. The patients were assigned to two groups, a control group with specific physiotherapy and the other group receiving the same specific kinetic treatment associated with the Mozart effect, for 2 hours daily, over 14 consecutive days. The patients were assessed using the Schwab \& England scale (for the degree of dependence) and the PHQ-9 scale (for depression) before and after therapy.

Results: After 2 weeks of therapy, the patients in the study group had much more obvious, statistically significant improvements in both scores.

Conclusions: Mozart's music, as an adjuvant intervention to specific kinetic treatment in patients with Parkinson's disease, had a beneficial effect, ameliorating the degree of disability and emotional status.

Key words: Parkinson's disease, neurorehabilitation, Mozart effect,

\section{Introduction}

Music, in addition to its socio-cultural role and recreational effect, seems to have a therapeutic effect, especially in potentiating attention and the concentration ability, and as a mood stabilizer (1-3). Mozart's music in particular caught the attention of research that started from empirical findings and small studies of its beneficial effect on the mentioned aspects, which led to the development of the concept of "Mozart effect". This was not unanimously accepted, being questioned by some authors (4). Subsequent studies discovered that Mozart's music influences the structure of brain wave bands, favoring the slow alpha and beta waves $(5,6)$. The beneficial effect of Mozart's music in neurological diseases was first demonstrated in children with treatment-refractory epilepsy, for which therapeutic alternatives were being searched and daily exposure to Mozart's music reduced the frequency of seizures by about $50 \%(7,8)$. Its use in neurorehabilitation was based on the expected effect on cognition and was initially applied to patients with dementia or cognitive decline after craniocerebral trauma, who had statistically significant improvements in specific evaluation scores (MMSE and ADS) compared to the control group $(3,9,10)$ having the support of the findings that the music is increasing the brain-derived neurotrophic factor (BDNF) and is modulating the nerve growth factor (NGF)(11). The effect in the rehabilitation of Parkinson's disease, including 
motor and non-motor symptoms (12), has been less studied, but starting from the beneficial effect in other neurodegenerative diseases (Alzheimer's dementia), and based on studies that demonstrate increased endogenous dopamine synthesis in the nigrostriatal system (11), this pilot study aims to evaluate the effect of Mozart's music associated with specific kinetic therapy as part of a rehabilitation program for patients with Parkinson's disease.

\section{Material and method}

A prospective observational study was conducted, which included 16 patients with Parkinson's disease attending a motor neurorehabilitation program for 14 consecutive days, through the "Power of Hope" project carried out at the "Saint Joseph" Medical Center Cluj-Napoca. The patients were divided into 2 groups of 8 patients each: the control group which received specific kinesiotherapy and occupational therapy for 2 hours daily, and the study group, for which Mozart's music was played in the background during the 2-hour kinesiotherapy and occupational therapy program. All patients gave their informed consent for participation. The inclusion criteria were age over 18 years, diagnosis of Parkinson's disease, Hoehn \& Yahr stages I-III, while the exclusion criteria were Parkinson-like syndrome or Parkinsonplus syndrome, Hoehn \& Yahr stages IV-V, associated diseases contraindicating kinesiotherapy. Each patient was assessed initially and at the end of the program using the following scales: the Schwab \& England ADL (Activities of Daily Living) scale which grades the functional status of patients with Parkinson's disease, the score decreasing in steps of $10 \%$ according to the degree of dependence (from $100 \%$ (normal) to $0 \%$ (most severe)) (13), and the PHQ-9 (Patient Health Questionnaire - 9) scale, an instrument useful to assess the presence of depression and its severity over the last 2 weeks by 9 questions with four answer variants (Not at all, Several days, More than half the days, Nearly every day) (14).
The specific physiotherapy program attended by both groups had as objectives the improvement of posture of the trunk and of the balance in standing and walking and, consequently, lowering the risk of falling. The methods of approaching consisted in dynamic exercises in order to increase the amplitude of the movements the scapulo-humeral belts and pelvic girdle, to strengthen postural muscles, to improve the gait (cadence walking, crossed walking, walking with trunk twists and with obstacles, with speed and direction changes; exercises on the wall bars and parallel bars with controlled lifting and imbalance and regaining of posture).

Mozart's compositions played for the study group were: "The Piano Sonata No 16 in C major", "Rondo Alla Turca", "Eine Kleine Nachtmusik", K.525, "Symphony No 35 in D major", K. 385 (Haffner Symphony), "Symphony No 40 in G minor", KV. 550, "The Magic Flute: Overture", "The Marriage of Figaro: Overture", "String Quartet No. 23 in F major, K. 590: I. Allegro moderato", "String Quartet No. 23 in F major, K. 590: IV. Allegro".

Statistical analysis was performed using Microsoft Excel. Categorical data were presented as diagrams, absolute and relative frequencies, and continuous variables were summarized using synthetic centrality or frequency histograms. Statistical analysis used the paired t test. A $p$ value lower than 0.05 allows rejection of the null hypothesis (absence of Mozart effect associated with kinesiotherapy in patients with Parkinson's disease) and acceptance of the alternative hypothesis (Mozart effect associated with kinesiotherapy in these patients).

\section{Results}

In Figure 1, which shows the distribution by age groups, it can be seen that both in the control group and the study group, patients aged between 61-70 years are predominant, representing $50 \%$ in both cases (Fig. 1) 


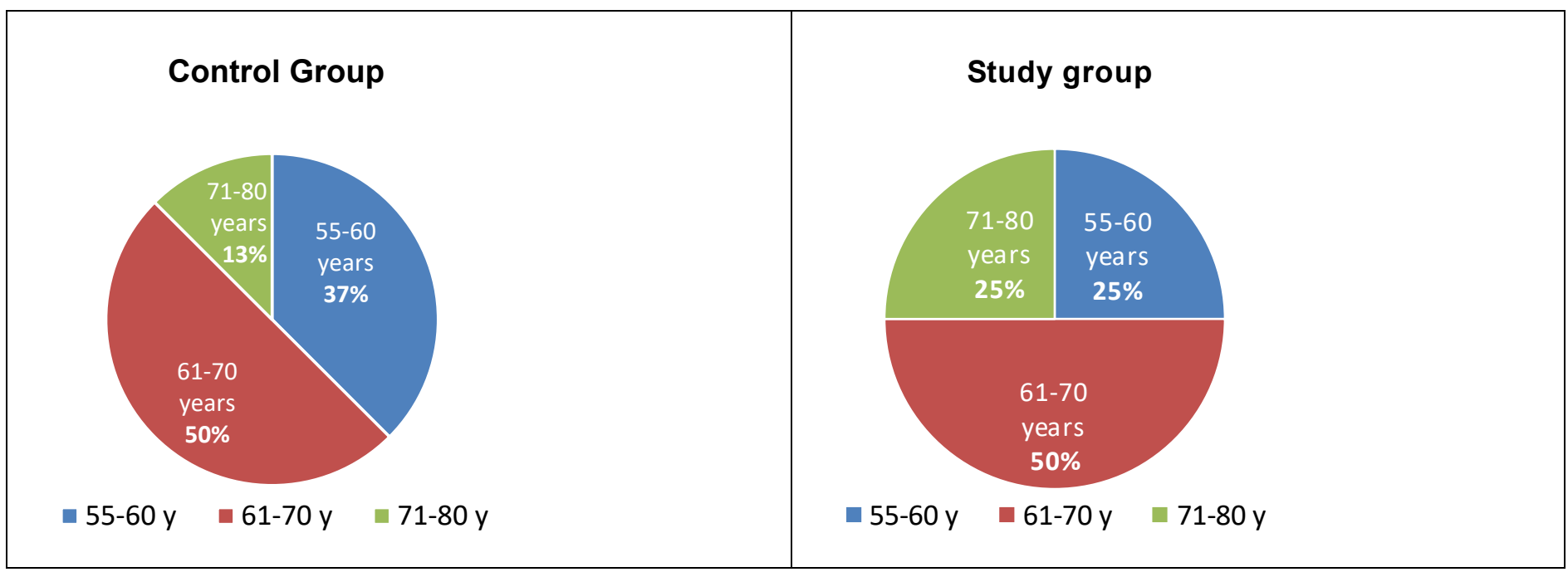

Fig. 1. Distribution of patients in the control and the study group depending on age

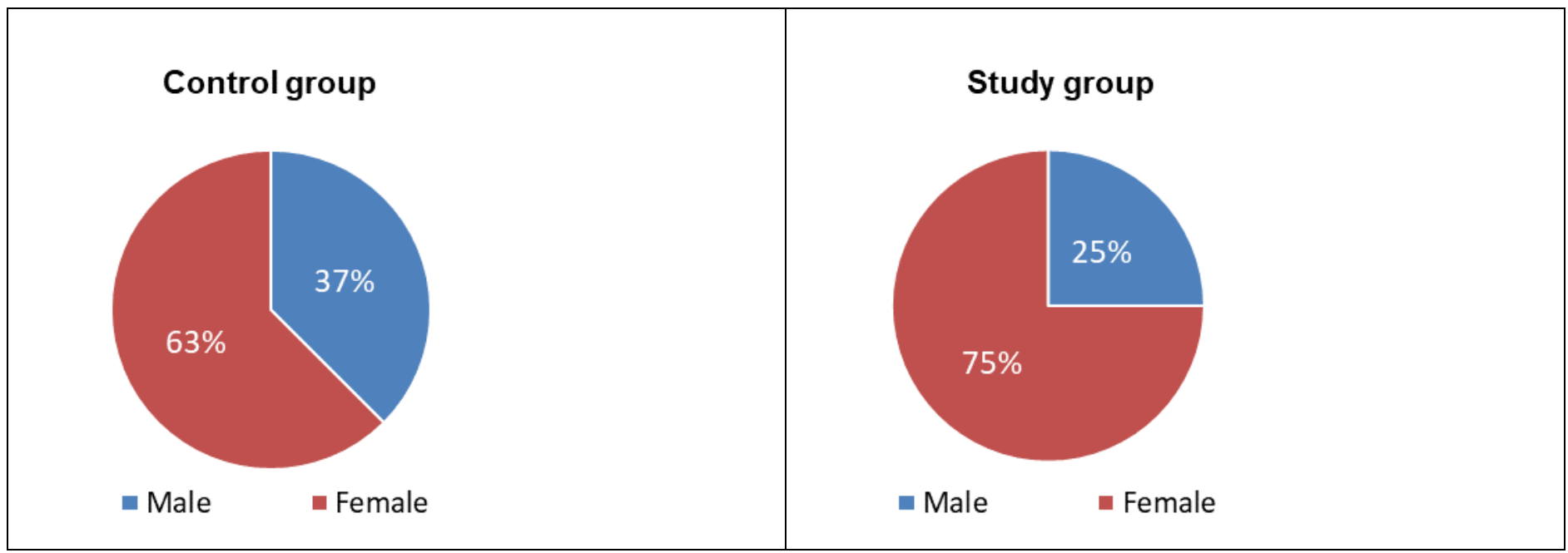

Fig. 2. Distribution of patients depending on sex

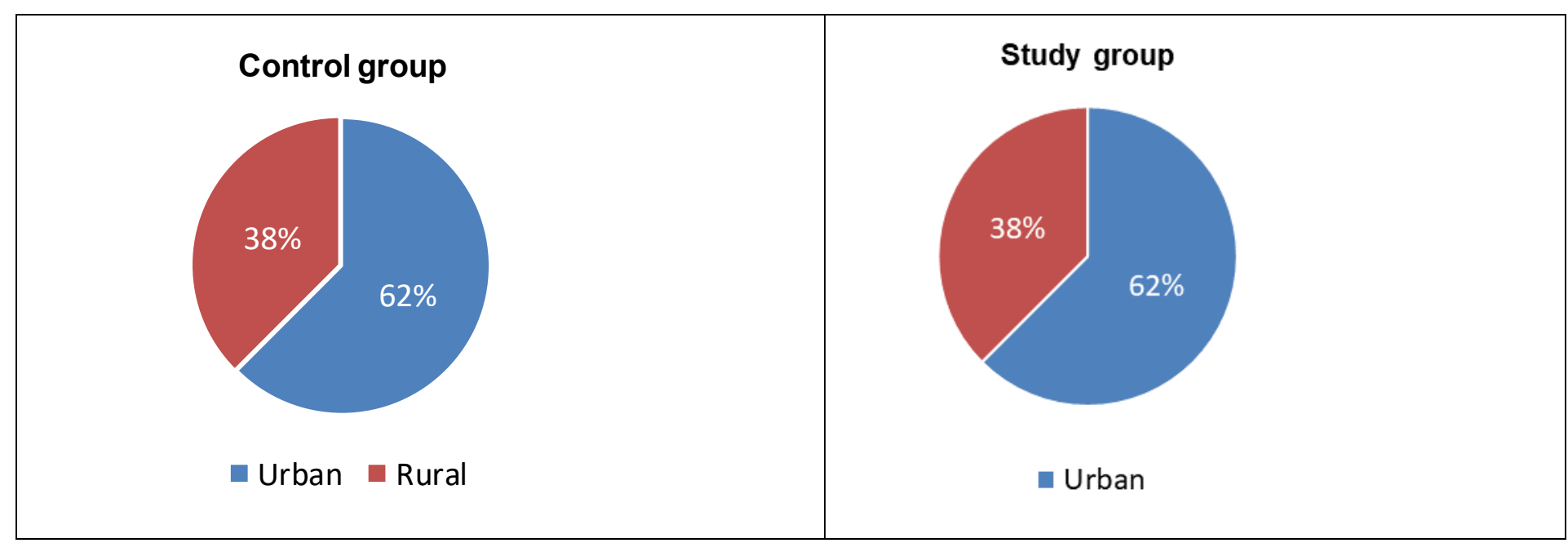

Fig. 3. Distribution of patients depending on the environment of origin 


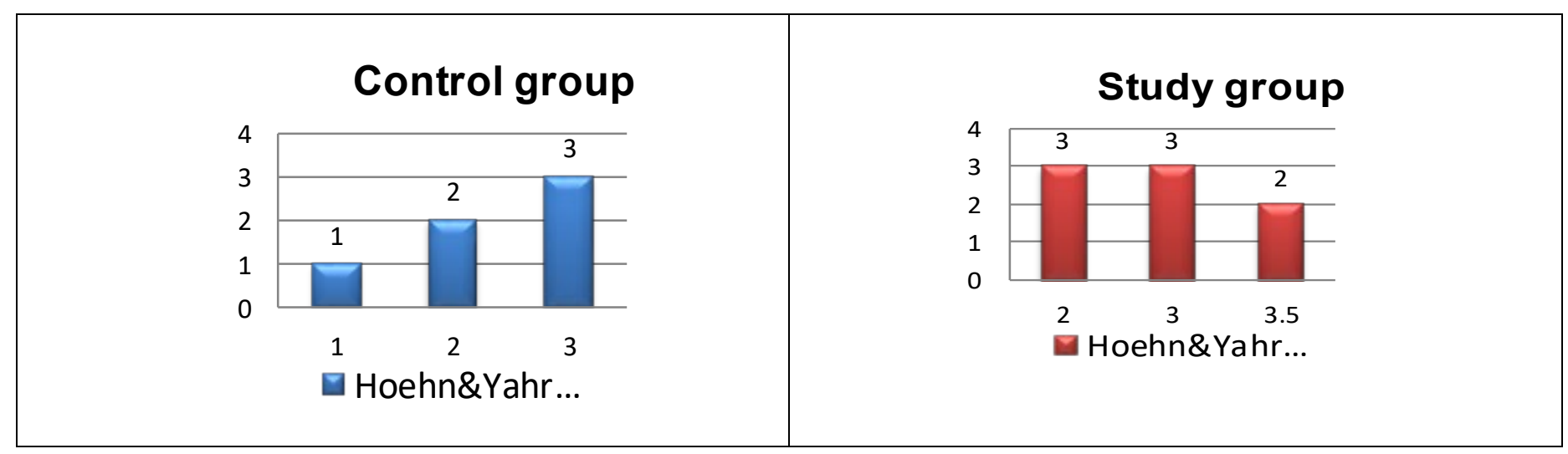

Fig. 4. Distribution of patients depending on disease staging according to the Hoehn\&Yahr scale

\begin{tabular}{|c|c|c|c|c|c|}
\hline \multicolumn{3}{c|}{ Schwab \& England } & \multicolumn{3}{c|}{ Schwab \& England } \\
\hline
\end{tabular}

Fig. 5. Schwab \& England score on day 14 compared to day 1 of therapy in both groups

The majority of the studied patients have stage III of the disease according to the Hoehn \& Yahr scale, and the mean score is higher in the study group (2.75) compared to the control group (2.34) (Fig. 4). Patient distribution depending on the score obtained following evaluation on the Schwab \& England scale on day 1 and day 14 of the neurorehabilitation program is found in Figure 5.

A comparison of the results between final values (day 14) and initial values (day 1) in both the control group and the study group shows an increase in the Schwab \& England score in both groups, with a more significant statistical improvement in the study group $(\mathrm{p}=0.001)$ compared to the control group $(\mathrm{p}=0.016)$.

Patients were assessed regarding the presence of anxious-depressive elements evidenced by applying the PHQ-9 test on days 1 and 14. Of the 9 items of the test, those mentioned below showed score changes, being analyzed in Figure 6:

- Loss of interest or pleasure in current activities

- Sleep disorders

- Fatigue or lack of energy

- Concentration difficulties

\section{Discussions}

In both groups, the highest proportion was represented by patients aged between 61 and 70 years. Also, in both groups the percentage of females was higher than that of males, although literature data support the predominance of men (15), and most came from rural areas.

The majority of the patients included in both groups had Hoehn \& Yahr stage III of the disease, with the mention that following random assignment of patients to the two groups, the mean score was higher in the study group (2.75) compared to the control group (2.34).

The Schwab \& England scale, reflecting the degree of disability/dependence in activities of daily living was statistically significantly improved in both the control and the study group, but in the latter case the improvement was highly statistically significant.

Of the 9 items of the PHQ-9 scale, 4 had changes reported by the patients: "loss of interest or pleasure in current activities" and "concentration 
difficulties", which registered a statistically significant improvement only in the study group, and "sleep disorders" and "fatigue or lack of energy", which were statistically significantly improved in both groups, with a more obvious improvement in the study group.
Loss of interest or pleasure in current activities PHQ-9

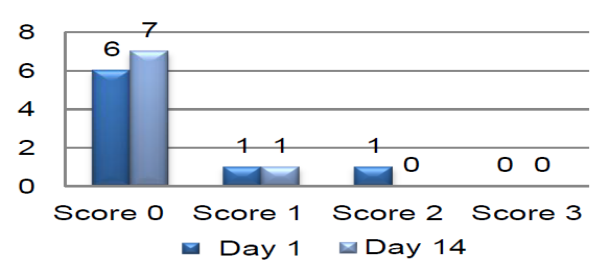

Control group: $p=0,085$

Sleeps disorders- PHQ-9

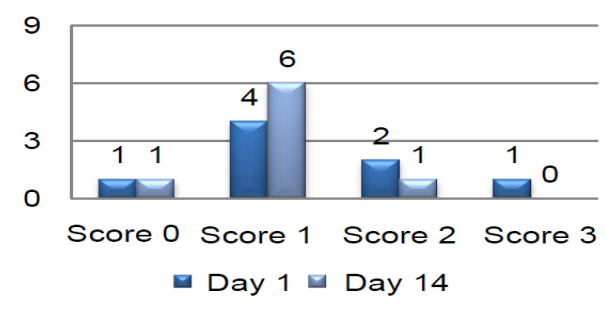

Control group: $\mathbf{p}=\mathbf{0 , 0 3 9}$

Fatigue or lack of energy - PHQ-9

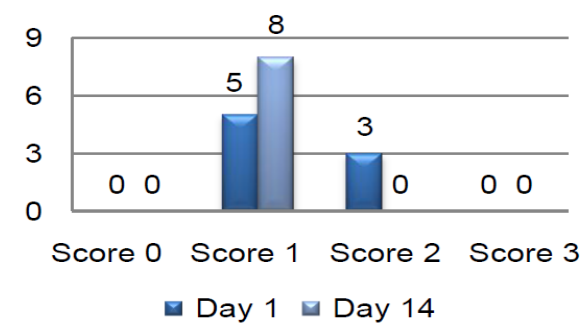

Control group: $\mathbf{p}=\mathbf{0 , 0 3 9}$

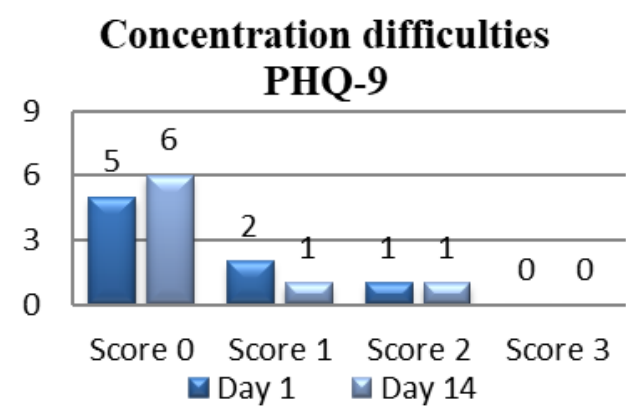

Control group: $\mathbf{p}=\mathbf{0 , 1 7 5}$

Fig. 6. Patient scores for the changed items, on the PHQ-9 test

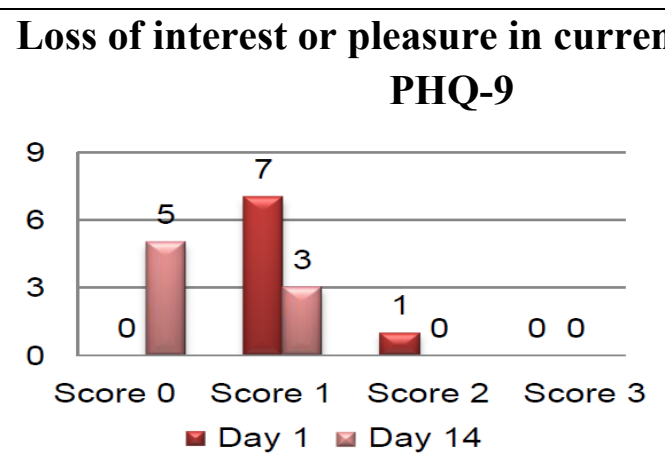

Study group: $\mathrm{p}=\mathbf{0 , 0 0 1}$

\section{Sleeps disorders- PHQ-9}

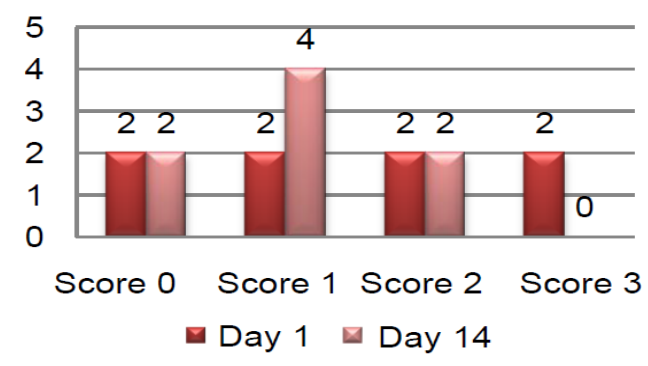

Study group: $p=0,016$

Fatigue or lack of energy - PHQ-9

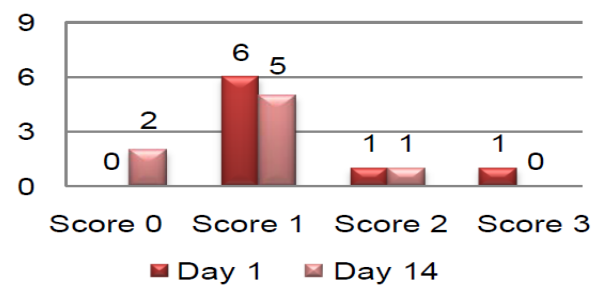

Study group: $p=0,016$

\section{Concentration difficulties}

$$
\text { PHQ-9 }
$$

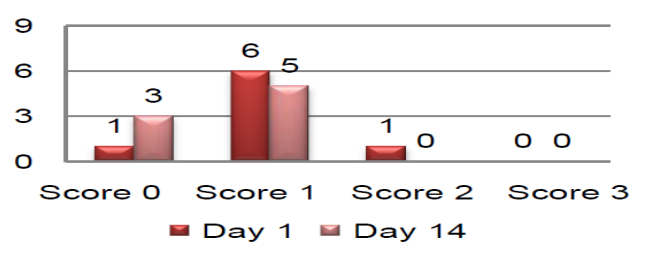




\section{Conclusion}

In our study, the association of Mozart's music with specific kinetic therapy in patients with Parkinson's disease potentiated the effect of rehabilitation therapy by decreasing the degree of disability reflected in the Schwab \& England score. It also had a mood-stabilizing role by reducing depressive elements, which was supported by the PHQ-9 score, ensuring increased compliance with the motor neurorehabilitation program and further potentiating its effect. It would be worth extending the study to larger socio-cultural categories of patients, in order to exclude possible bias related to the preference for or even acquaintance with certain types of music. The study suggests that a minimal intervention such as the association of music with rehabilitation therapy can make a notable difference in results.

\section{References}

1. Trimble M, Hesdorffer D. Music and the brain: the neuroscience of music and musical appreciation. BJPsych international. 2017;14(2):28-31.

2. Arias Gomez M. [Music and neurology]. Neurologia. 2007;22(1):39-45.

3. Thaut MH. The future of music in therapy and medicine. Annals of the New York Academy of Sciences. 2005;1060:303-8.

4. Pietschnig J, Voracek M, Formann AK. Mozart effect-Shmozart effect: A meta-analysis. Intelligence. 2010;38(3):314-23.

5. Modi V. Impact of Music on Attention among Patients with Parkinson's Disease EC Psychology and Psychiatry. 2018;7(6):321-9.

6. Jenkins JS. The Mozart effect. Journal of the Royal Society of Medicine. 2001;94(4):170-2.

7. Grylls E, Kinsky M, Baggott A, Wabnitz C, McLellan A. Study of the Mozart effect in children with epileptic electroencephalograms. Seizure. 2018;59:77-81.

8. Hughes JR, Daaboul Y, Fino JJ, Shaw GL. The "Mozart effect" on epileptiform activity. Clin Electroencephalogr. 1998;29(3):109-19.
9. Van de Winckel A, Feys H, De Weerdt W, Dom R. Cognitive and behavioural effects of musicbased exercises in patients with dementia. Clinical rehabilitation. 2004;18(3):253-60.

10. Park S, Williams RA, Lee D. Effect of Preferred Music on Agitation After Traumatic Brain Injury. Western journal of nursing research. 2016;38(4):394-410.

11. Angelucci F, Ricci E, Padua L, Sabino A, Tonali PA. Music exposure differentially alters the levels of brain-derived neurotrophic factor and nerve growth factor in the mouse hypothalamus. Neuroscience letters. 2007;429(2-3):152-5.

12. Dogaru G, Stanescu I. Treatment and Rehabilitation in Non-Motors Symptoms of Parkinson's Disease. Balneo Research Journal. 2014:188-92.

13. Marinus J, Visser M, Stiggelbout AM, Rabey JM, Martinez-Martin P, Bonuccelli U, et al. A short scale for the assessment of motor impairments and disabilities in Parkinson's disease: the SPES/SCOPA. Journal of neurology, neurosurgery, and psychiatry. 2004;75(3):388-95.

14. Kroenke K, Spitzer RL, Williams JB. The PHQ9: validity of a brief depression severity measure. Journal of general internal medicine. 2001;16(9):606-13.

15. Miller IN, Cronin-Golomb A. Gender differences in Parkinson's disease: clinical characteristics and cognition. Movement disorders : official journal of the Movement Disorder Society. 2010;25(16):2695-703. 